\title{
Effect of the stress-strain state on the path of quasi-cleavage hydrogen-assisted cracking in low-carbon steel
}

\author{
E. D. Merson ${ }^{\dagger, 1}$, V. A. Poluyanov ${ }^{1}$, P. N. Myagkikh ${ }^{1}$, D. L. Merson ${ }^{1}$, A. Yu. Vinogradov² \\ †Mersoned@gmail.com
}

${ }^{1}$ Institute of Advanced Technologies, Togliatti State University, Togliatti, 445667, Russia

${ }^{2}$ Department of Mechanical and Industrial Engineering, Norwegian University of Science and Technology - NTNU, Trondheim, N-7491 Norway

Conflicting data on the effect of the stress-strain state on the shape and path of quasi-cleavage cracks in hydrogen-embrittled steels and iron have been reported in the literature recently. However, this issue is important for understanding the nature of hydrogen embrittlement (HE) and, particularly, the role of hydrogen in the mechanism of crack propagation. In this regard, in the present study, smooth and notched specimens of low-carbon steel were tensile tested with in-situ cathodic hydrogen charging. The side surface of the specimens fractured in this way was examined by scanning electron microscopy aiming at quantitative characterization of the length and curvature of secondary side surface cracks. In addition, fractographic analysis was performed. A great number of cracks having a characteristic S-shaped curved geometry are found on the side surface of the notched specimens. Moreover, the cracks can smoothly curve both on the scale of several grains as well as within the individual grain interior. In contrast, in the case of smooth specimens, the cracks generally exhibit relatively straight appearance. Quantitative analysis using a statistically-representative dataset has shown that, on average, the curvature of cracks in the notched specimens is significantly higher and is varied over a wider range of values than in the smooth specimens. Based on the data obtained, it is concluded that the path of quasi-cleavage cracks in hydrogen-embrittled ferritic and ferritepearlitic low-carbon steels is mainly determined by the characteristics of the stress-strain state, and not by the microstructure or crystallographic orientation of individual grains.

Keywords: hydrogen embrittlement, quasi-cleavage, cracks, steel.

УДК: 538.911

\section{Влияние напряженно-деформированного состояния на путь распространения трещин квазискола в низкоуглеродистой стали, охрупченной водородом}

\author{
Мерсон Е. Д. ${ }^{\dagger, 1}$, Полуянов В. А. ${ }^{1}$, Мягких П.Н. ${ }^{1}$, Мерсон Д. Л. ${ }^{1}$, Виноградов А. Ю. ${ }^{2}$ \\ ${ }^{1}$ Научно-исследовательский институт прогрессивных технологий, Тольяттинский государственный университет, \\ Тольятти, 445667, Россия \\ ${ }^{2}$ Кафедра механики и промышленной инженерии, Норвежский технологический университет - НТУ, \\ Тронхейм, N-7491, Норвегия
}

В литературе существуют противоречивые сведения о влиянии напряженно-деформированного состояния на геометрию и путь трещин квазискола в охрупченных водородом сталях и железе. Данный вопрос является важным с точки зрения понимания природы водородной хрупкости (BX) и, в частности, роли водорода в механизме распространения трещин. В связи с этим в настоящей работе гладкие и надрезанные образцы низкоуглеродистой стали были испытаны на растяжение в процессе электролитического наводороживания. После разрушения образцов их боковая поверхность была исследована при помощи сканирующей электронной микроскопии с целью количественного анализа длины и кривизны вторичных трещин. Кроме того, был проведен фрактографический анализ. Установлено, что на боковой поверхности образцов с надрезом присутствует большое количество трещин с характерной S-образно искривленной геометрией. Причем плавное искривление трещин может происходить 
как в масштабе нескольких зерен, так и внутри тела зерна. В то же время в случае гладких образцов, трещины в основном имеют относительно прямолинейную форму. При помощи количественного анализа с репрезентативным набором данных показано, что в среднем кривизна трещин в образцах с надрезом существенно выше и варьируется в более широком диапазоне значений, чем в гладких образцах. На основе полученных данных сделан вывод, о том, что путь трещин квазискола в охрупченных водородом ферритных и феррито-перлитных низкоуглеродистых сталях главным образом определяется характером напряженно-деформированного состояния, а не микроструктурой или кристаллографической ориентацией отдельных зерен.

Ключевые слова: водородная хрупкость, квазискол, трещины, сталь.

\section{1. Введение}

Растворяясь в железе и сталях, атомарный водород снижает напряжения, необходимые для зарождения и распространения трещин, что в свою очередь вызывает ухудшение механических свойств, главным образом, пластичности и прочности $[1,2]$. Данное явление, называемое водородной хрупкостью (BX), может приводить к внезапным разрушениям стальных деталей и является актуальной проблемой для многих отраслей промышленности. В частности, в настоящий момент в большинстве развитых стран ведется активная работа по разработке и созданию инфраструктуры для водородной энергетики, где низкоуглеродистые стали планируется использовать для изготовления трубопроводов и хранилищ газообразного водорода. В связи с этим, в последнее время наблюдается повышенный интерес научного и инженерного сообщества к вопросам создания научно-обоснованных принципов дизайна микроструктуры сталей с повышенной стойкостью к ВХ [3-5], а также разработки моделей, позволяющих производить расчет восприимчивости сталей к воздействию водорода $[6,7]$. Однако работа в данном направлении во многом затруднена по причине недостаточного понимания механизмов BX. Разрушение наводороженных низкоуглеродистых сталей с ферритной и феррито-перлитной структурой, как правило, сопровождается образованием поверхности разрушения с морфологией квазискола, отличающейся большим разнообразием элементов вязкого разрушения: гребней отрыва, нано-ямок и др. [8-12]. Специфика внешнего вида и количественные характеристики таких изломов позволяют утверждать, что они принципиально отличаются от изломов истинного скола, образующихся обычно в этих материалах, например, при хладноломкости $[10,13]$. Существует две прямо противоположных точки зрения относительно природы формирования поверхности квазискола. Согласно одной из них квазискол является результатом распространения трещины скола, реализации которого способствует водород, например, путем ослабления межатомных связей в кристаллической решетке [14], либо за счет блокирования эмиссии дислокаций из устья трещины [15]. При этом наличие следов вязкого разрушения в изломе объясняется влиянием пластической деформации, сопровождающей процесс роста трещины. Другая точка зрения заключается в том, что рельеф квазискола может быть сформирован в результате вязкого разрушения $[11,16,17]$. Имеются сведения о том, что водород может облегчать подвижность дислокаций [18]. Таким образом, предпо- лагается, что в обогащенном водородом локальном микрообъеме вблизи вершины трещины происходит пластификация материала и разрушение осуществляется по видоизмененному вязкому механизму посредством образования и слияния микро- и нано-пор $[11,16,17]$. Стоит заметить, что описанные выше механизмы, предполагают принципиально разный путь распространения трещин. Известно, что скол в ОЦК железе и сталях с ферритной структурой осуществляется вдоль строго определенных кристаллографических плоскостей, преимущественно, семейства $\{100\}$ [19]. Тогда, как вязкое разрушение не привязано жестко к кристаллографии. В наших предыдущих работах было показано, что в низкоуглеродистой стали фасетки квазискола имеют существенно меньший средний угол разориентировки, по сравнению с фасетками истинного скола [10,13]. Данный факт свидетельствует о том, что путь трещин квазискола преимущественно осуществляется вне плоскостей истинного скола. Кроме того, в ряде работ было показано, что в отличие от трещин истинного скола, трещины квазискола могут сильно искривляться внутри зерна [20-22], что предположительно происходит под действием неоднородных полей напряжений и деформаций, возникающих, например, вблизи надреза. Наличие надреза в образце приводит, во-первых, к изменению в прилегающей к надрезу области напряженнодеформированного состояния от простого (линейного) к сложному, а, во-вторых, к проявлению эффекта концентрации напряжений, т.е. к резкому возрастанию действующих напряжений вблизи их концентратора (надреза). При этом траектория развития трещины является следствием реализации как сингулярной, так и несингулярной составляющей (параметр локального стеснения деформаций у вершины трещины) действующих вблизи трещины или надреза напряжений [23]. Однако в литературе существуют противоречивые сведения о влиянии надреза на путь распространения трещин квазискола. Так, в работе [21] было показано, что в наводороженном железе S-образно искривленные трещины квазискола образуются как в присутствии надреза, так и без него. Поэтому авторы пришли к выводу, что искривленная форма трещин не связана с влиянием надреза. В то же время, в одной из наших предыдущих работ [22], было обнаружено, что искривление трещин квазискола происходит главным образом вблизи надреза, тогда как вдали от него трещины имеют относительно прямолинейную геометрию. В связи с существующими в литературе противоречиями целью настоящего исследования было установить влияние поля напряжений от надреза на геомет- 
рию и путь распространения трещин квазискола в низкоуглеродистой стали. В отличие от предыдущих работ, в настоящем исследовании для описания геометрии трещин был применен количественный анализ, основанный на статистически-репрезентативной выборке.

\section{2. Материал и методика исследования}

Гладкие и надрезанные образцы для испытаний на растяжение с геометрией, показанной на Рис. 1a и b, соответственно, были вырезаны из горячекатаного листа промышленной низкоуглеродистой стали марки S235JR, химический состав которой представлен в Табл. 1. Данная сталь также была использована в качестве модельного материала в наших предыдущих работах $[10,13,22]$. Для создания равновесной феррито-перлитной микроструктуры, предварительно отшлифованные образцы отжигали в вакууме при температуре $950^{\circ} \mathrm{C}$ в течение 1 часа. Средний размер зерна после такой термообработки составляет 36 мкм (6-7 балл зерна по ISO 643-2019).

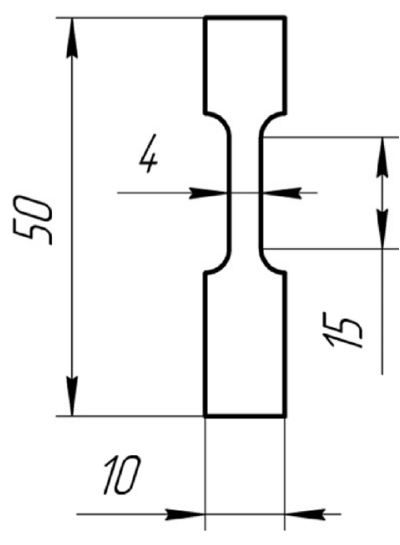

a
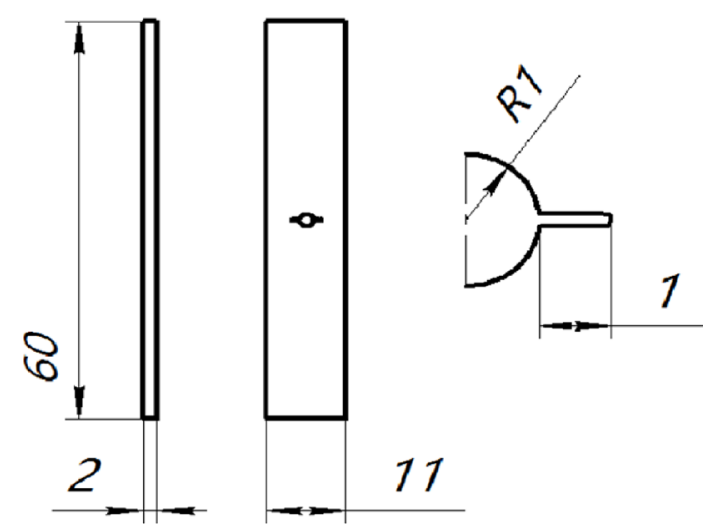

$\mathrm{b}$

Рис. 1. Геометрия гладких (a) и надрезанных (b) образцов.

Fig. 1. Geometry of smooth (a) and through-notched (b) specimens.
Финишная подготовка поверхности отожженных образцов включала полировку при помощи суспензий с частицами 3, 1 и 0.25 мкм с последующим травлением в растворе $3 \% \mathrm{HNO}_{3}$ в ультразвуковой ванне.

Испытание на растяжение производили при помощи разрывной машины AG-X plus ф. Shimadzu при скорости движения траверсы 0.1 мм/мин. На протяжении всего процесса растяжения производилось электролитическое in-situ наводороживание образца. Для этого перед началом испытания на образце закреплялась электрохимическая ячейка с платиновым анодом в виде спирали. Ячейку заполняли электролитом состава $5 \% \mathrm{H}_{2} \mathrm{SO}_{4}+1.5$ г/л тиомочевины, и непосредственно в момент начала растяжения подавали постоянный ток плотностью $5 \mathrm{~mA} / \mathrm{cm}^{2}$. Испытание останавливали при полном разрушении образцов.

Боковую поверхность и изломы образцов исследовали при помощи сканирующего электронного микроскопа (CЭM) JEOL JCM-6000. Для оценки кривизны вторичных трещин $C$ использовали формулу (1):

$$
C=\frac{\left(l_{1}+l_{2}\right) / 2}{l_{3}}
$$

где $l_{1}$ и $l_{2}$ - длины двух берегов трещины, a $l_{3}$ - расстояние между двумя вершинами трещины. В каждом образце было измерено не менее 100 трещин.

\section{3. Результаты и обсуждение}

На Рис. 2 показаны общий вид и микростроение изломов испытанных образцов. Поверхность разрушения обоих образцов представлена исключительно морфологией квазискола, характерными признаками которой являются: (1) гребни отрыва, ориентированные преимущественно вдоль направления роста трещины, (2) полосы, ориентированные перпендикулярно направлению роста трещины, и (3) относительно плоские участки между гребнями и полосами. Таким образом, все трещины, обнаруженные на поверхности образцов, являются трещинами квазискола.

На Рис. 3 представлены снимки боковой поверхности надрезанных и гладких образцов после разрушения. Как видно, под изломом на боковой поверхности обоих образцов присутствует множество вторичных транскристаллитных трещин. У образца с надрезом, трещины наблюдаются только в области пластической зоны вблизи надреза. Тогда как у образца без надреза вся поверхность рабочей части покрыта трещинами. Таким образом, по-видимому, существенная пластическая деформация является необходимым условием образования трещин квазискола в отожженной низкоуглеродистой стали. В случае образца с надрезом большое количество трещин имеет характерную искривленную S-образную

Табл. 1. Химический состав стали S235JR.

Table 1. Chemical composition of the steel S235JR.

\begin{tabular}{|c|c|c|c|c|c|c|c|c|c|c|}
\hline Элемент / Element & $\mathrm{C}$ & $\mathrm{Cu}$ & $\mathrm{Si}$ & $\mathrm{Mn}$ & $\mathrm{P}$ & $\mathrm{S}$ & $\mathrm{Cr}$ & $\mathrm{Ni}$ & $\mathrm{Al}$ & $\mathrm{Fe}$ \\
\hline Bec.\% / Wt.\% & 0.129 & 0.067 & 0.02 & 0.42 & 0.019 & 0.015 & 0.05 & 0.007 & 0.028 & Ocнова / Balance \\
\hline
\end{tabular}




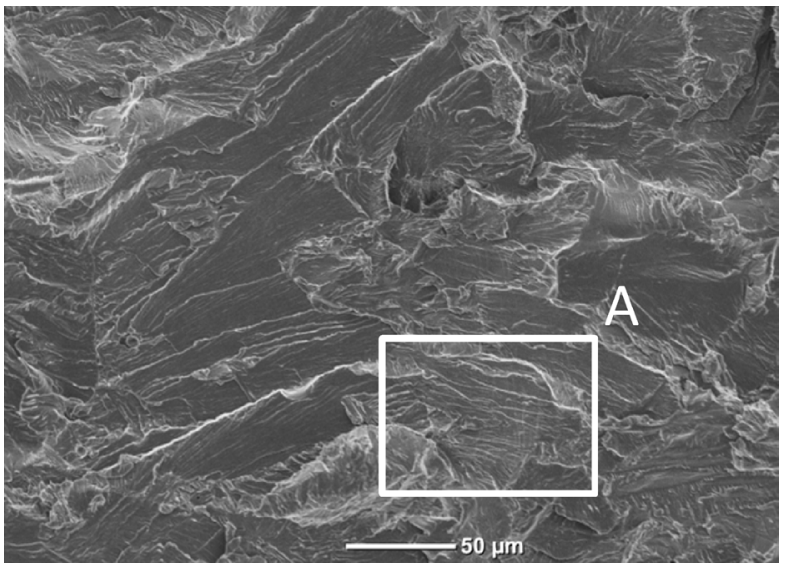

a

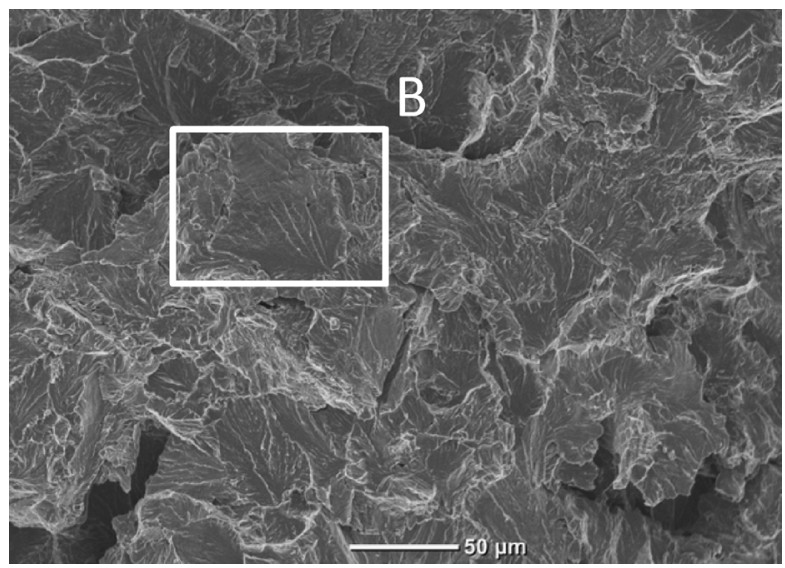

c

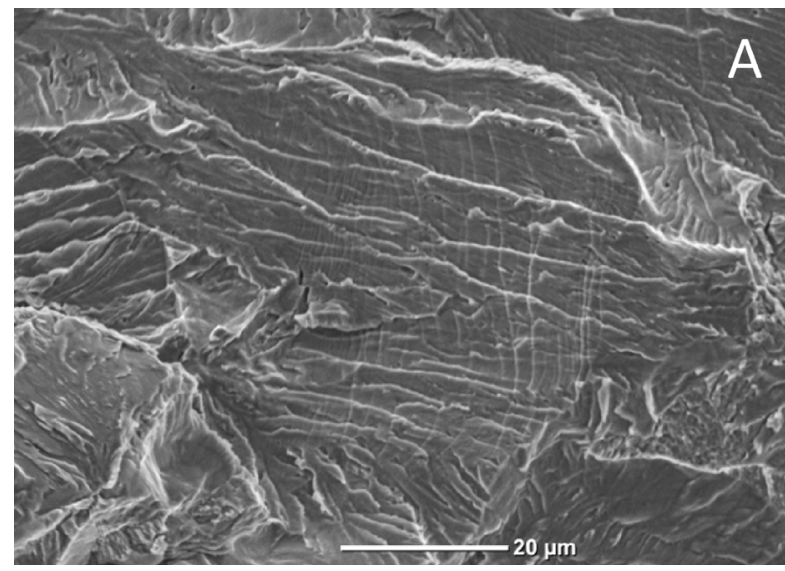

$\mathrm{b}$

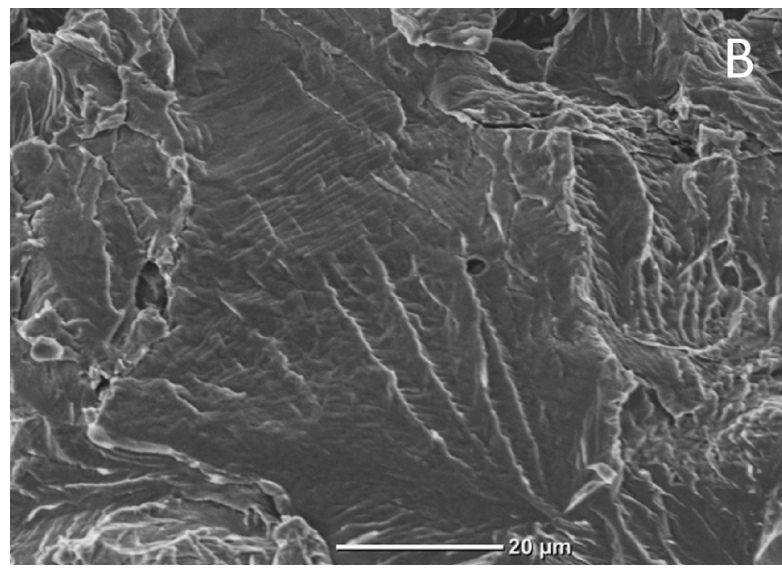

$\mathrm{d}$

Рис. 2. Морфология изломов гладких (a, b) и надрезанных (c, d) образцов.

Fig. 2. The fracture surface morphology of the smooth (a, b) and the notched (c, d) specimens.

форму (Рис. $3 \mathrm{~d}, \mathrm{f})$, которая была также отмечена в других работах $[21,22]$. При этом зачастую трещины имеют искривленную форму даже в масштабе одного зерна (Рис. 3f), что свидетельствует о слабой привязке пути трещин к каким-либо конкретным кристаллографическим плоскостям. В случае гладкого образца, трещины, в основном, ориентированы перпендикулярно к оси растяжения (Рис. 3 a). При этом они имеют гораздо более прямолинейную геометрию (Рис. 3c), по сравнению с теми, которые обнаружены в образце с надрезом. Стоит, однако, отметить, что даже такие относительно прямые трещины в микромасштабе демонстрируют характерную волнистость (Рис. 3 е).

Хотя в обоих случаях встречаются, как искривленные, так и относительно прямые трещины, в образце без надреза доля прямых трещин существенно больше, чем в образце с надрезом. Об этом свидетельствуют результаты количественного анализа геометрии трещин. Так, на Рис. 4 представлены гистограммы распределения значений кривизны трещин для образцов без надреза и с надрезом, соответственно. Из них видно, что кривизна трещин в образце с надрезом варьируется в гораздо более широком диапазоне, чем в гладком образце. При этом в среднем кривизна трещин в образце с надрезом заметно выше, чем в образце без надреза (см. значения $C$, приведенные на гистограммах на Рис. 4). Следовательно, результаты настоящего исследования подтверждают сделанное ранее предположение о том, что поле повышенных напряжений и изменение напряженно-деформированного состояния вблизи надреза оказывают сильное и, по-видимому, определяющее влияние на путь трещин квазискола в наводороженной стали. Данное влияние таково, что даже внутри зерна распространение трещин квазискола может происходить по плавно искривленной траектории. Эта особенность указывает на принципиальное отличие механизма их роста, от механизма роста трещин истинного скола, путь которых в масштабе ферритного зерна жестко привязан к кристаллографическим плоскостям семейства $\{100\}$. Внутри зерен в сталях трещины истинного скола всегда распространяются прямолинейно, либо меняют направление роста под углом $90^{\circ}$. В то же время, если распространение трещины происходит путем слияния микропор, то искривление ее пути внутри зерна под действием поля напряжений видится вполне оправданным.

Следует отметить, что результаты настоящего исследования расходятся с результатами работы [21], авторы которой пришли к выводу, что наличие надреза в наводороженных образцах чистого железа не оказывает влияния на кривизну трещин квазискола. Однако данный вывод был сделан авторами лишь 


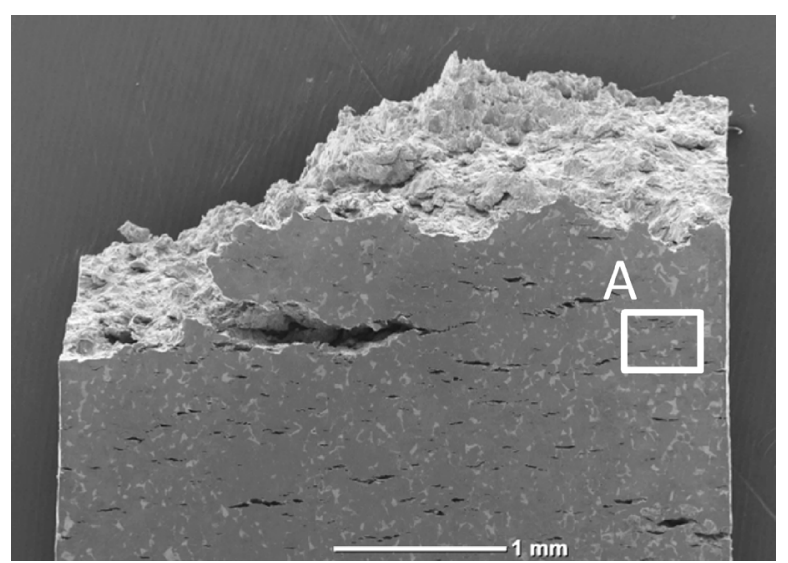

a

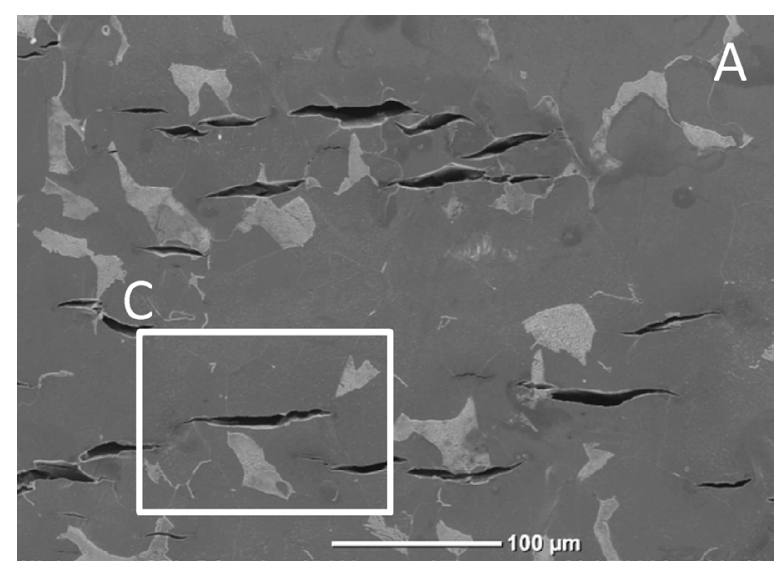

c

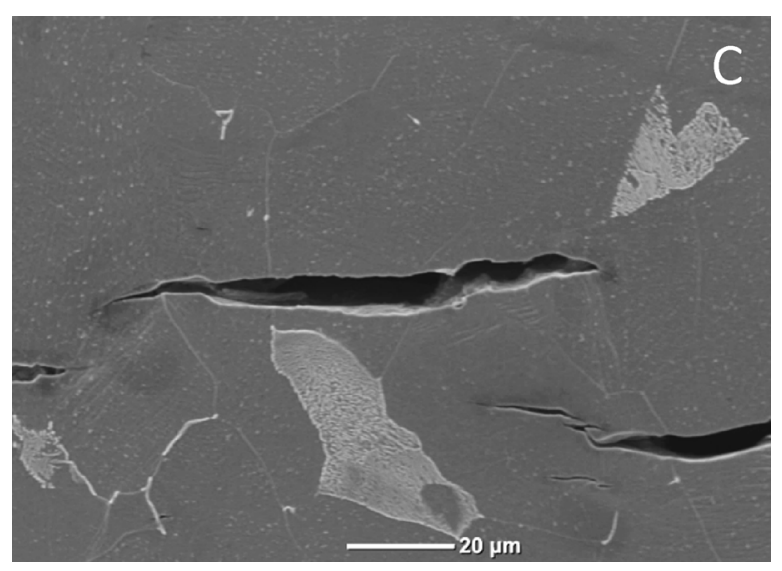

e

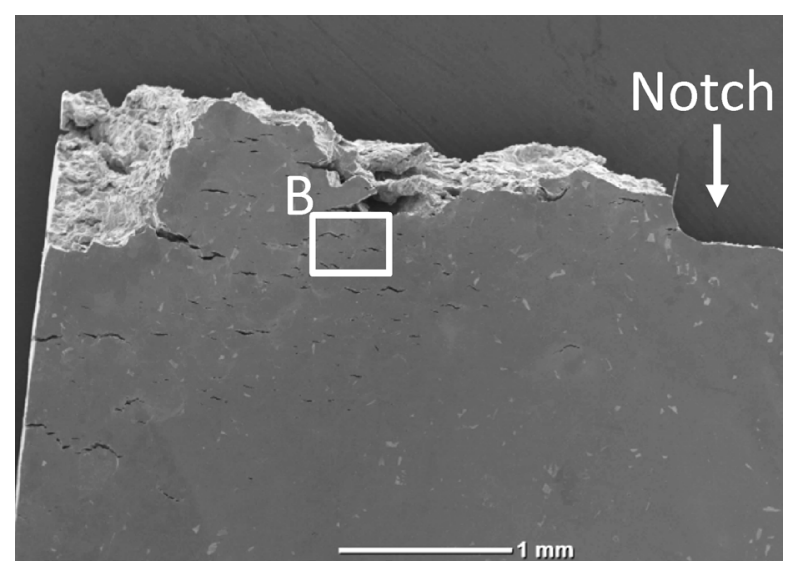

b

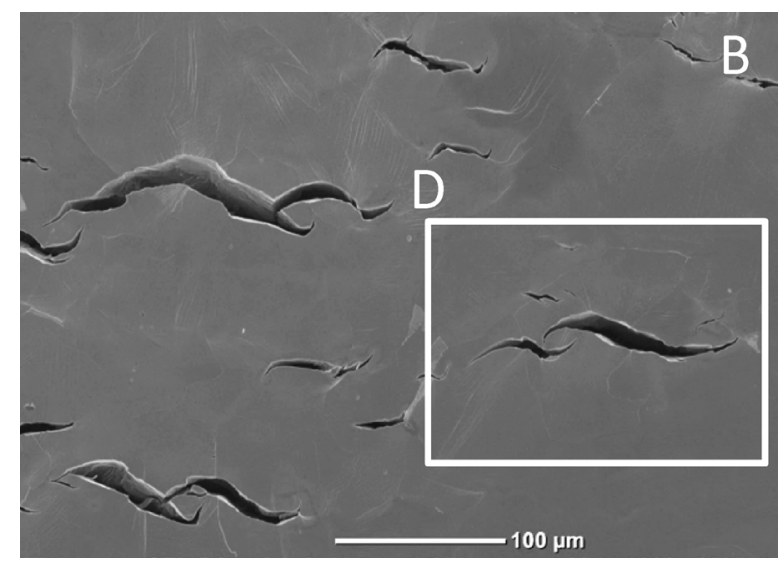

d

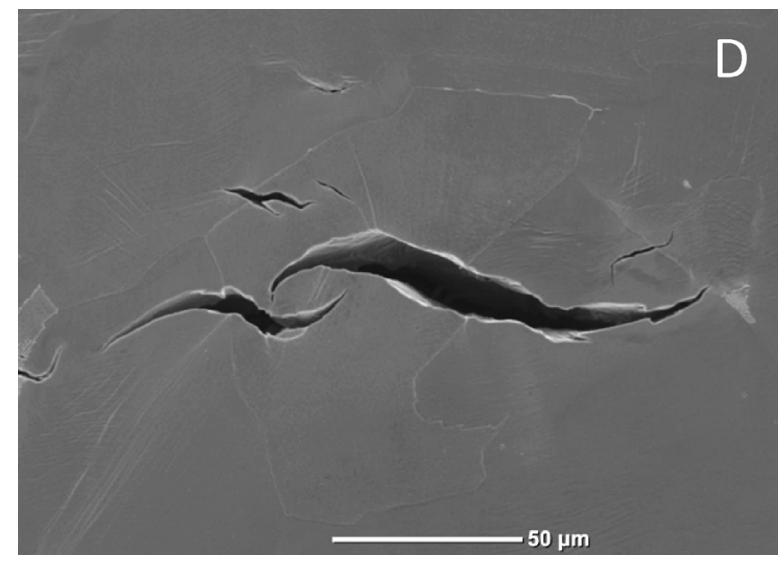

f

Рис. 3. Снимки боковой поверхности гладкого (a, c, e) и надрезанного (b, d, f) образцов после испытания при in-situ наводороживании. Fig. 3. Side surface of the smooth (a, c, e) and the notched (b, d, f) specimens tensile tested under in-situ hydrogen charging.

на основании того, что S-образные трещины были обнаружены, как в надрезанном, так и в гладком образцах. При этом количественный анализ геометрии трещин с репрезентативной выборкой, такой, как в настоящей работе, проведен не был. В данной работе тоже показано, что искривленные трещины, действительно, присутствуют и в гладком, и в надрезанном образцах. Однако при помощи количественного анализа достоверно установлено, что искривленных трещин в образце с надрезом существенно больше. Следовательно, наличие надреза в наводороженных низкоуглеродистых сталях способствует искривлению вторичных трещин квазискола.

\section{4. Выводы}

1. Поле напряжений и вид напряженно-деформированного состояния являются главными факторами, определяющим путь трещин квазискола в наводороженной низкоуглеродистой стали, в том числе, в масштабе одного ферритного зерна.

2. Согласно данным количественного анализа геометрии трещин, наличие надреза в образце наводороженной стали приводит к искривлению трещин квазискола.

3. Распространение трещин квазискола в низкоуглеродистой стали, не осуществляется по механизму истинного скола. 


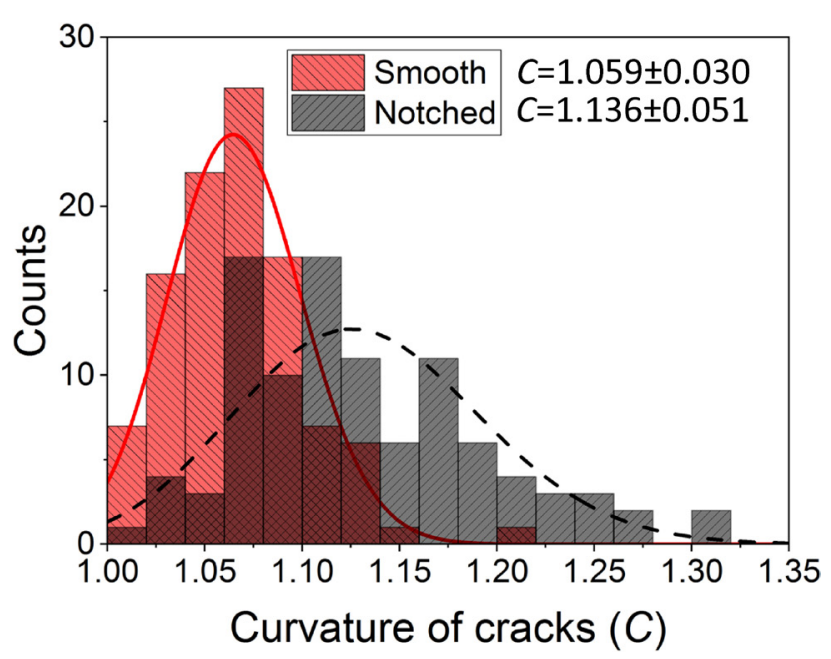

Puc. 4. (Color online) Гистограммы распределения значений кривизны вторичных трещин на боковой поверхности гладкого и надрезанного образцов. В верхнем правом углу гистограммы приведены средние значения кривизны трещин $C$ для двух типов образцов.

Fig. 4. (Color online) The distributions of the cracks' curvature values for the smooth and the notched specimens. On the right upper corner the average values of cracks' curvature are provided for the two types of the specimens.

Благодарности / Aknowledgements. Работа выполнена при финансовой поддержке Российского научного фонда (nроекm 19-79-00188). / Financial support from the Russian Science Foundation through the grant-in-aid No. 19-79-00188 is gratefully appreciated.

\section{Литература/References}

1. S. P. Lynch. Corros. Rev. 30, 63 (2012). Crossref

2. I. M. Robertson, P. Sofronis, A. Nagao, M. L. Martin, S. Wang, D. W. Gross, K. E. Nygren. Metall. Mater. Trans. A. 46, 2323 (2015). $\underline{\text { Crossref }}$

3. M. Asadipoor, A. Pourkamali Anaraki, J. Kadkhodapour, S. M. H. Sharifi, A. Barnoush. Mater. Sci. Eng. A. 772, 138762 (2020). $\underline{\text { Crossref }}$

4. T. I. Ramjaun, S. W. Ooi, R. Morana, H. K. D. H. Bhadeshia. Mater. Sci. Technol. 34, 1737 (2018). Crossref
5. B. Ozdirik, T. Depover, L. Vecchi, K. Verbeken, H. Terryn, I. De Graeve. J. Electrochem. Soc. 165, 787 (2018). Crossref

6. T. Das, E. Legrand, S. V. Brahimi, J. Song, S. Yue. Eng. Fract. Mech. 224, 1 (2020). Crossref

7. A. Arora, H. Singh, D. K. Mahajan. Mater. Sci. Eng. A. 787, 139488 (2020). Crossref

8. T. Homma, S. Anata, S. Onuki, K. Takai. Tetsu-ToHagane/Journal Iron Steel Inst. Japan. 106, 651 (2020). $\underline{\text { Crossref }}$

9. K. Okada, A. Shibata, Y. Takeda, N. Tsuji. Int. J. Hydrogen Energy. 43, 11298 (2018). Crossref

10. E. Merson, A. V. Kudrya, V.A. Trachenko, D. Merson, V. Danilov, A. Vinogradov. Mater. Sci. Eng. A. 665, 35 (2016). Crossref

11. M.L. Martin, J.A. Fenske, G.S. Liu, P. Sofronis, I. M. Robertson. Acta Mater. 59, 1601 (2011). Crossref

12. E.D. Merson, P.N. Myagkikh, G.V. Klevtsov, D. L. Merson, A. Vinogradov. Eng. Fract. Mech. 210, 342 (2019). Crossref

13. E.D. Merson, V.A. Poluyanov, P.N. Myagkikh, D. L. Merson, A. Y. Vinogradov. Lett. Mater. 10 (3), 303 (2020). (in Russian) [Е.Д. Мерсон, В.А. Полуянов, П.Н. Мягких, Д.Л. Мерсон, А.Ю. Виноградов. Письма о материалах. 10 (3), 303 (2020).] Crossref

14. X. Chen, W. W. Gerberich. Metall. Trans. A. 22, 59 (1991). Crossref

15. D. Birenis, Y. Ogawa, H. Matsunaga, O. Takakuwa, J. Yamabe, Ø. Prytz, A. Thøgersen. Mater. Sci. Eng. A. 756, 396 (2019). Crossref

16. S. P. Lynch. Acta Metall. 36, 2639 (1988). Crossref

17. T. Neeraj, R. Srinivasan, J. Li. Acta Mater. 60, 5160 (2012). Crossref

18. M.L. Martin, M. Dadfarnia, A. Nagao, S. Wang, P. Sofronis. Acta Mater. 165, 734 (2019). Crossref

19. P. A. Davies, M. Novovic, V. Randle, P. Bowen. J. Microsc. 205, 278 (2002). Crossref

20. M. L. Martin, I. M. Robertson, P. Sofronis. Scr. Mater. 59, 3680 (2011). Crossref

21. A. Laureys, T. Depover, R. Petrov, K. Verbeken. Mater. Sci. Eng. A. 690, 88 (2017). Crossref

22. E.D. Merson, P.N. Myagkikh, V.A. Poluyanov, D. L. Merson, A. Vinogradov. Eng. Fract. Mech. 214, 177 (2019). Crossref

23. M. Hadj Meliani, Yu. G. Matvienko, G. Pluvinage. Int. J. of Fracture. 167, 173 (2011). $\underline{\text { Crossref }}$ 